University of Wollongong

Research Online

Faculty of Engineering - Papers (Archive)

Faculty of Engineering and Information

Sciences

$1-2-2006$

\title{
A 70 Kiloparsec X-Ray Tail in the Cluster A3627
}

M. Sun

Michigan State University, USA

C. Jones

Harvard-Smithsonian Center for Astrophysics, Cambridge, USA

P. Nulsen

University of Wollongong

M. Donahue

Michigan State University, USA

G. M. Voit

Michigan State University, USA

Follow this and additional works at: https://ro.uow.edu.au/engpapers

Part of the Engineering Commons

https://ro.uow.edu.au/engpapers/292

\section{Recommended Citation}

Sun, M.; Jones, C.; Nulsen, P.; Donahue, M.; and Voit, G. M.: A 70 Kiloparsec X-Ray Tail in the Cluster A3627 2006.

https://ro.uow.edu.au/engpapers/292

Research Online is the open access institutional repository for the University of Wollongong. For further information contact the UOW Library: research-pubs@uow.edu.au 


\title{
A 70 KILOPARSEC X-RAY TAIL IN THE CLUSTER A3627
}

\author{
M. Sun, ${ }^{1}$ C. Jones, ${ }^{2}$ W. Forman, ${ }^{2}$ P. E. J. Nulsen, ${ }^{2,3}$ M. Donahue, ${ }^{1}$ and G. M. Voit ${ }^{1}$ \\ Received 2005 November 16; accepted 2005 December 20; published 2006 January 18
}

\begin{abstract}
We present the discovery of a $70 \mathrm{kpc}$ X-ray tail behind the small late-type galaxy ESO 137-001, in the nearby, hot $(T=6.5 \mathrm{keV})$ merging cluster A3627, from both Chandra and XMM-Newton observations. The tail has a length-to-width ratio of $\sim 10$. It is luminous $\left(L_{0.5-2 \mathrm{keV}} \sim 10^{41} \mathrm{ergs} \mathrm{s}^{-1}\right)$, with a temperature of $\sim 0.7 \mathrm{keV}$ and an X-ray $M_{\text {gas }}$ of $\sim 10^{9} M_{\odot}(\sim 10 \%$ of the galaxy's stellar mass $)$. We interpret this tail as the stripped interstellar medium of ESO 137-001 mixed with the hot cluster medium, with this blue galaxy being converted into a gaspoor galaxy. Three X-ray point sources are detected in the axis of the tail, which may imply active star formation there. The straightness and narrowness of the tail also imply that the turbulence in the intracluster medium is not strong on scales of $20-70 \mathrm{kpc}$.
\end{abstract}

Subject headings: galaxies: clusters: general — galaxies: clusters: individual (A3627) — galaxies: individual (ESO 137-001) - X-rays: galaxies

\section{INTRODUCTION}

The intracluster medium (ICM) has long been proposed to play a vital role in shaping the properties of cluster galaxies, especially through the stripping of cold galactic material. Ram pressure stripping is likely an important factor for galaxy transformation in rich environments (see, e.g., Gunn \& Gott 1972; Quilis et al. 2000). Radio observations have long revealed an $\mathrm{H}$ I deficiency in cluster galaxies, which indicates stripping (e.g., Giovanelli \& Haynes 1985). In X-rays, the stripped tails of late-type galaxies only began to be revealed by Chandra and XMM-Newton data (Wang et al. 2004; Sun \& Vikhlinin 2005, hereafter SV05; Machacek et al. 2005). Recently, we carried out a systematic analysis of the X-ray thermal coronae of $\sim 200$ galaxies in 25 nearby $(z<0.05)$, hot $(k T>3 \mathrm{keV})$ clusters (M. Sun et al. 2006, in preparation). During our analysis, we found an X-ray tail in A3627, which is apparently associated with the late-type galaxy ESO 137-001. The narrowness and length of the tail make it the most dramatically stripped X-ray tail of a galaxy found to date.

A3627 is a nearby massive galaxy cluster in the core of the Great Attractor. Kraan-Korteweg et al. (1996) found, in a deep imaging and spectroscopic survey for galaxies behind the Milky Way, that A3627 (at a Galactic latitude of -7.2) is an extremely rich cluster, rivaling Coma and Perseus in mass and galactic content. A major cluster merger is implied by earlier X-ray observations from the Röntgensatellit (ROSAT) and the Advanced Satellite for Cosmology and Astrophysics that reveal a southeast-northwest cluster elongation (Fig. 1a) and a temperature gradient $(5-8 \mathrm{keV})$ along the same direction (Böhringer et al. 1996; Tamura et al. 1998).

The radial velocity of ESO 137-001 is $4630 \pm 58 \mathrm{~km} \mathrm{~s}^{-1}$, almost the same as that of A3627 $\left(4707 \mathrm{~km} \mathrm{~s}^{-1}\right.$, from the NASA/IPAC Extragalactic Database [NED]). In this Letter, we adopt the cluster redshift to calculate the distance of ESO 137001. Assuming $H_{0}=70 \mathrm{~km} \mathrm{~s}^{-1} \mathrm{Mpc}^{-1}, \Omega_{M}=0.3$, and $\Omega_{\Lambda}=$ 0.7 , the luminosity distance is $68.2 \mathrm{Mpc}$ and $1^{\prime \prime}=0.321 \mathrm{kpc}$. Uncertainties quoted in this Letter are $1 \sigma$.

\footnotetext{
${ }^{1}$ Department of Physics and Astronomy, Michigan State University, East Lansing, MI 48824; sunm@pa.msu.edu.

${ }^{2}$ Harvard-Smithsonian Center for Astrophysics, 60 Garden Street, Cambridge, MA 02138.

${ }^{3}$ On leave from School of Engineering Physics, University of Wollongong, Wollongong, NSW 2522, Australia.
}

\section{CHANDRA AND XMM-NEWTON DATA}

The tailed X-ray source lies in a $15 \mathrm{ks}$ Chandra observation of A3627 (ObsID 4956) performed with the Advanced CCD Imaging Spectrometer (ACIS) on 2004 June 14-15. No background flares were detected. Standard data analysis has been performed. The calibration files used correspond to Chandra Calibration Database version 3.1.0.

The source is also contained within an $18 \mathrm{ks}$ XMM-Newton observation from 2004 August 12. The data were reduced with the Science Analysis System, 6.5.0. After removing large background flares, clean exposures of $17.8,18.0$, and $12.1 \mathrm{ks}$ remain for the MOS1, MOS2, and PN CCDs, respectively.

\section{THE TAILED X-RAY SOURCE}

The XMM-Newton photon image and adaptively smoothed Chandra images are shown in Figures $1 b-1 d$, which clearly show a long, narrow tail. The tail is only visible in the soft $\mathrm{X}$-ray images. Its brighter southeast end is on the cluster galaxy ESO 137-001 (Fig. 1c). There are no other galaxies as bright as ESO 137-001 in the $B$ band within $7^{\prime}$ of ESO 137-001 in the direction of the tail. Thus, we conclude that the extended X-ray source is physically associated with ESO 137-001.

ESO 137-001 is in a crowded field with many Galactic stars. The galaxy is bright in the optical $B$ band but faint in the Two Micron All Sky Survey $K_{s}$ band. The $B$-band luminosity is (2.3-4.0) $\times 10^{10} L_{B, \odot}$ from NED and HyperLeda, depending on the correction for internal extinction. The $K_{s}$-band luminosity is $1.4 \times 10^{10} L_{K, \odot}$. Assuming a $K_{s}$-band mass-to-light ratio of $0.73(M / L)_{\odot}$ (Cole et al. 2001), the total stellar mass of ESO $137-001$ is only $1.1 \times 10^{10} M_{\odot}$, which makes it $\sim 5$ times less massive than an $L_{*}$ galaxy. The $B-K_{s}$ color of ESO 137-001 is 1.0-1.6, much smaller than the $B-K_{s}$ color of a typical early-type or late-type galaxy (3.7-4.0 and $\sim 3$, respectively; Jarrett 2000). Thus, ESO 137-001 is a very blue galaxy. Spectroscopic studies (Woudt et al. 1999, 2004) have revealed $\mathrm{H} \alpha, \mathrm{H} \beta,[\mathrm{O} \mathrm{III}] \lambda 5007$, and [N II] $\lambda 6584$ emission lines in the spectrum of ESO 137-001. Thus, we conclude that ESO 137001 is a late-type galaxy with active star formation.

\subsection{The Diffuse X-Ray Emission}

To better quantify the properties of the tail, we measured Chandra surface brightness profiles along and across it 

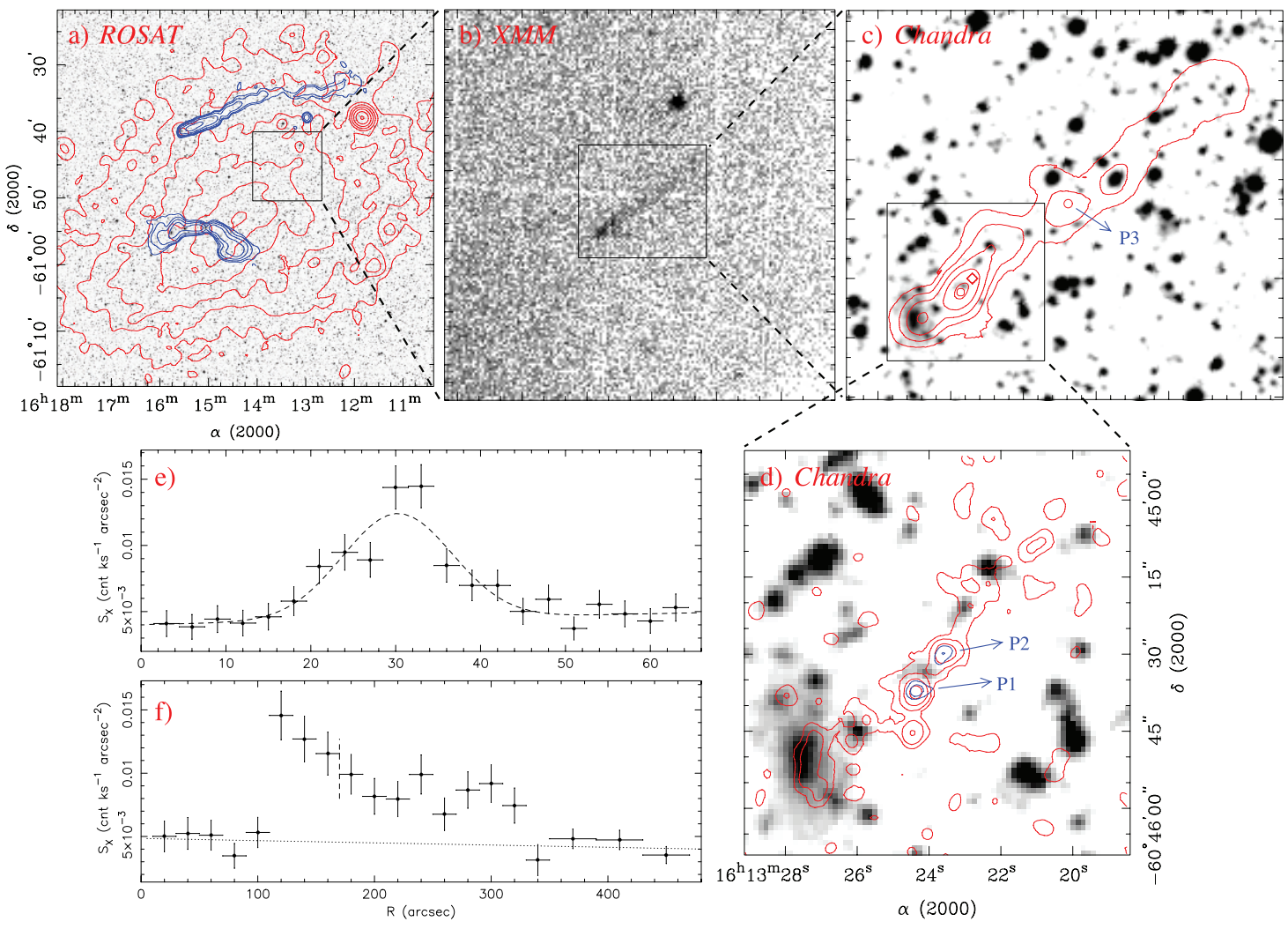

FIG. 1.-(a) ROSAT contours (red) for A3627 superposed on the Digitized Sky Survery (DSS) image (gray). The 843 MHz contours of two strong radio sources in A3627 (blue) are also shown (from the Sydney University Molonglo Sky Survey). The cluster is in the Galactic plane, so the foreground star density is high. (b) XMM-Newton 0.5-2 keV photon image (PN and MOS combined, 11'.1 $\times 11^{\prime} 1$ ) of the boxed region in $(a)$. A source with a narrow-angle tail, pointing in a similar direction to the radio tailed source, is significant. $(c)$ Zoom in on $(b)(3.7 \times 3.3)$. Chandra 0.5-2 keV contours are superposed on the DSS II image. The Chandra image (background-subtracted and exposure-corrected) has been adaptively smoothed. The head of the X-ray tail is on the galaxy ESO 137-001. P3 is a point source. $(d)$ Zoom in on $(c)(1 ; 3 \times 1 ' 3)$. The X-ray tail is shown by the red Chandra $0.5-2 \mathrm{keV}$ contours on the DSS II image. The 2-6 keV contours of two point sources (P1 and P2) are shown in blue. (e) Chandra $0.5-2 \mathrm{keV}$ surface brightness profile across the tail (from southwest to northeast, with a length of 3.7). The dashed line is a fit to the profile with a Gaussian and background. $(f)$ Chandra $0.5-2 \mathrm{keV}$ surface brightness profile along the direction of the tail (from southeast to northwest, with a width of $25^{\prime \prime}$ ). The dotted line is the fit to the local background, while the dashed vertical line marks the position where we separate "head" and "trail" regions in the spectral analysis. The nucleus of the galaxy is at $R=115$ ".

(Figs. $1 e$ and $1 f$ ). The surface brightness has been corrected for background (from the blank-sky background) and exposure. There is a sharp edge at its southeast end, with a brightness jump of a factor of $\sim 3$. The tail is detected to $3: 7$ (or $71 \mathrm{kpc}$ ) northwest of ESO 137-001. The width of the tail is $\$ 25^{\prime \prime}$ (or $8 \mathrm{kpc}$ ). If we fit the profile across the tail with a Gaussian plus the local background, the derived full width at half-maximum is only $15.2_{-2.1}^{+2.3}$ arcsec. Thus, this feature is very narrow, with a length-to-width ratio of $\sim 10$.

Integrated Chandra and XMM-Newton spectra of the source were extracted from a rectangular region centered on the source $\left(3 ! .7 \times 30^{\prime \prime}\right.$ for Chandra and $4{ }^{\prime} .1 \times 50^{\prime \prime}$ for $\left.X M M\right)$. We were forced to use a larger extraction region for $X M M$, as the width of the tail is comparable to the half-power diameter of the $X M M$ mirror $\left(15^{\prime \prime}\right)$. Background was taken from surrounding regions, a $4.5 \times 2^{\prime}$ box for Chandra and a $5^{\prime} \times 2.5$ box for XMM, excluding the source region. Within the source region, $230 \pm$ 24 (point sources excluded), $967 \pm 95$, and $872 \pm 103$ net counts in the $0.5-2 \mathrm{keV}$ band are collected from the ACIS, $\mathrm{PN}$, and MOS instruments, respectively. The current data do not allow us to constrain the absorption. As the spectra are well fitted with Galactic absorption at low energies, we fixed the absorption at the Galactic value, $2 \times 10^{21} \mathrm{~cm}^{-2}$. The solar photospheric abundance table from Anders \& Grevesse (1989) is adopted. A lower energy cutoff of $0.5 \mathrm{keV}$ is used to minimize the calibration uncertainties at low energies. The upper energy cutoff is $7 \mathrm{keV}$. Each spectrum was fitted with a MEKAL model. The abundance is very low, but the gas temperature is not sensitive to abundance (e.g., $T=0.66 \pm$ $0.06 \mathrm{keV}$ if $Z=1 Z_{\odot}$ ). All spectra showed evidence for a hard X-ray excess, so a power-law component was included. Inclusion of the hard component lowers the gas temperature by $10 \%-20 \%$ but greatly improves the fit. The nature of this hard X-ray component is unclear. The expected unresolved contribution from active galactic nuclei (AGNs) is small. There may be some residual emission from the hot ICM, either mixed in the tail or due to the uncertain normalization of the background. Emission of unresolved X-ray binaries in the tail (see $\S 3.2$ ) is another possibility. Regardless of the model used for the hard component, hot ICM (MEKAL), X-ray binaries, or AGNs (power law), the results for the gas properties are almost the same. The Chandra and XMM results are consistent, so we fitted them simultaneously. The gas temperature is $\sim 0.7 \mathrm{keV}$ (Table 1). We also derived temperatures in two regions, "head" and "trail," defined in Figure $1 f$. The trail is only marginally hotter $(1 \sigma)$ than the head. There are some discrepancies between the derived Chandra and XMM luminosities, at the level of $20 \%-33 \%$, which may be due to uncertainties in the back- 
TABLE 1

Results of SPECTRAL Fits

\begin{tabular}{|c|c|c|c|c|c|}
\hline Region $^{\mathrm{a}}$ & Instrument & Model $^{\mathrm{b}}$ & Parameters $^{\mathrm{c}}$ & $L_{0.5-2 \mathrm{keV}}{ }^{\mathrm{d}}$ (gas) & $L_{0.5-2 \mathrm{keV}}{ }^{\mathrm{d}}$ (hard) \\
\hline Total & Chandra/XMM & MEKAL+PL & $T=0.69 \pm 0.08 \mathrm{keV}, Z=0.05_{-0.02}^{+0.05}$ solar & 8.6 (ACIS), 12 (PN), 10 (MOS) & 1.2 (ACIS), $5.7(X M M)$ \\
\hline Head & Chandra/XMM & MEKAL + PL & $T=0.64_{-0.22}^{+0.08} \mathrm{keV}, Z=0.04_{-0.03}^{+0.05}$ solar & 4.0 (ACIS), 5.5 (PN), 4.7 (MOS) & 1.0 (ACIS), $2.1(X M M)$ \\
\hline Trail & Chandra/XMM & MEKAL+PL & $T=0.83_{-0.11}^{+0.13} \mathrm{keV}, Z=0.16_{-0.10}^{+0.16}$ solar & 4.4 (ACIS), 5.7 (PN), 5.4 (MOS) & 0.7 (ACIS), $3.6(X M M)$ \\
\hline Point sources ...... & Chandra & PL & $\Gamma=1.6 \pm 0.4$ & $\ldots$ & 1.3 \\
\hline ........... & Chandra & MEKAL & $T=6.3_{-0.7}^{+1.1} \mathrm{keV}, Z=0.1_{-0.1}^{+0.2}$ solar & $\ldots$ & $\ldots$ \\
\hline
\end{tabular}

${ }^{a}$ See Fig. 1 for the definition of the head and trail regions. For Chandra analysis of diffuse emission, three point sources are excluded, while they are unresolved in the XMM-Newton data. The Chandra spectra of these three point sources and the surrounding ICM region ( $\$ 3.2)$ are also analyzed.

${ }^{\mathrm{b}}$ There is a hard excess in each spectrum of the tail, so a power-law (PL) model (with a fixed photon index of 1.7) is included in the fits for the total, head, and trail regions.

${ }^{c}$ Galactic absorption $\left(2 \times 10^{21} \mathrm{~cm}^{-2}\right)$ is adopted for all spectral analyses. The Cash statistic is used for all spectra except that of the ICM. The fits are all very good.

${ }^{\mathrm{d}}$ The $0.5-2 \mathrm{keV}$ luminosity (in units of $10^{40} \mathrm{ergs} \mathrm{s}^{-1}$ ) of the gas and the hard component.

ground normalization (especially for $X M M$, where the background is higher).

The X-ray gas mass in the tail is estimated by assuming a $25^{\prime \prime} \times 25^{\prime \prime} \times 1$ '. 1 cylinder for the head and a 2'.6 long cylinder for the trail with constant density. From the normalization derived from the MEKAL fits, the average electron densities in the head and the trail are $(1.8-3.5) \times 10^{-2} f^{-1 / 2} \mathrm{~cm}^{-3}$ and $(0.7-$ 1.7) $\times 10^{-2} f^{-1 / 2} \mathrm{~cm}^{-3}$, respectively, while the $\mathrm{X}$-ray gas mass is $(0.7-1.3) \times 10^{9} f^{1 / 2} M_{\odot}$ and $(0.6-1.5) \times 10^{9} f^{1 / 2} M_{\odot}$, respectively, where $f$ is the filling factor of the X-ray gas. If the abundance in the head is 0.3 times solar (the value in the trail within $1 \sigma$ ), the average electron density and the X-ray gas mass of the head drop by $40 \%$. The filling factor may be large if the observed tail is really due to mixing of cold clouds and hot ICM (see § 4). Since the galaxy and cluster radial velocities are nearly the same, the projected length of the tail may be comparable to the actual length. Thus, the total X-ray gas mass is on the order of $10^{9} M_{\odot}$.

The temperature of the surrounding ICM (a $3^{\prime} \times 6^{\prime}$ box, excluding the tailed source) was also measured. Since A3627 is behind the Galactic plane, the Galactic soft X-ray foreground is strong. This is proved by the 3-5 times higher than nominal flux in the R4-R5 band of the ROSAT All-Sky Survey image at the position of A3627. As there is no local background available, we used the blank-sky background and added a $0.2 \mathrm{keV}$ MEKAL component (unabsorbed) to mimic the soft $\mathrm{X}$-ray excess. The derived ICM temperature is $6.3_{-0.7}^{+1.1} \mathrm{keV}$ (Table 1). The error from the uncertainty in the temperature of the soft excess is included, as we allow it to vary from 0.1 to $0.4 \mathrm{keV}$ (Markevitch et al. 2003). The ICM electron density from the ROSAT data (Böhringer et al. 1996) is $\sim 1.4 \times 10^{-3} \mathrm{~cm}^{-3}$ at the projected position of ESO 137-001. Within the uncertainties, the thermal pressure is the same inside and outside the tail, especially if the abundance is not that low.

\subsection{X-Ray Point Sources in the Tail}

There are three Chandra point sources right on the axis of the tail (P1-P3 in Fig. 1), with a total of 73 counts in the 0.5$10 \mathrm{keV}$ band. Their combined spectrum is hard (Table 1). Within 3' of ESO 137-001, only an additional, fainter point source is detected. The possibility of having three background AGNs aligned in the narrow tail for this shallow observation is $\sim 0.03 \%$, based on the AGN number density derived from three Chandra pointings toward A3627. If P1-P3 are located in the tail, $L_{2-10 \mathrm{kev}}=2.7 \times 10^{40} \mathrm{ergs} \mathrm{s}^{-1}$ (the total), which is similar to the total $L_{\mathrm{X}}$ of three ultraluminous X-ray sources in UGC 6697 (SV05). Since the ICM pressure cannot move stars, the most likely scenario is that there is active massive star formation in the tail, as the stripped clouds, away from their main heating source (the stellar UV radiation), can cool. $\mathrm{H} \alpha$ imaging and $\mathrm{H} \mathrm{I}$ data are required in order to test this hypothesis.

\section{DISCUSSION}

The X-ray tail of ESO 137-001 is unique. Our systematic study covers a sky area of $3.1 \mathrm{deg}^{2}$ in 25 nearby hot clusters, but only UGC 6697 in A1367 has a similar tail. However, UGC 6697 is 8 times more luminous than ESO 137-001 in the $K_{s}$ band, and its tail does not extend significantly outside of the galaxy (SV05). Even the stripped X-ray tails known in cool clusters and groups (e.g., Machacek et al. 2005) are not as dramatic as the tail of ESO 137-001. The X-ray tail of ESO 137-001 is only the third known X-ray tail of a late-type galaxy in a hot cluster (after Wang et al. [2004] and SV05). While its proximity enables further detailed studies, the tail length and the X-ray point sources in the tail provide additional insight.

Long and narrow X-ray tails have been seen in simulations by Stevens et al. (1999) when the stripping happens gradually and gas is replenished in the galaxy. Similar H I tails have also been seen in other simulations (e.g., Quilis et al. 2000; Vollmer et al. 2001). Two $75 \mathrm{kpc} \mathrm{H} \alpha$ trails have been detected behind two small late-type galaxies in A1367 (Gavazzi et al. 2001), but no X-ray tails are seen in the XMM-Newton data. A $110 \mathrm{kpc}$, but tilted, $\mathrm{H}$ I tail was observed from NGC 4388 in Virgo (Oosterloo \& van Gorkom 2005). The tail of ESO 137-001 cannot be the accretion wake of ESO 137-001, as the galaxy is too small and the ICM is too hot. The ESO 137-001 tail could be either the stripped X-ray gas of ESO 137-001 or cold material from ESO 137-001 (e.g., H I gas) mixed with hot ICM. The first scenario requires that the stripped tenuous X-ray gas survive all kinds of instabilities and yet remain distinct from the surrounding ICM for $\sim 10^{8} \mathrm{yr}$, which is about the time needed for ESO 137-001 to travel from the end of the tail to its current position (71 kpc in projection) at a velocity of $1000 \mathrm{~km} \mathrm{~s}^{-1}$ (the cluster radial velocity dispersion is $897 \mathrm{~km} \mathrm{~s}^{-1}$ ). Mixing of cold interstellar medium (ISM) with hot ICM is preferred, as it is expected after the stripping of cold ISM. The average temperature after mixing $(\sim 0.7 \mathrm{keV})$ is $\left(M_{\mathrm{ICM}} T_{\mathrm{ICM}}+M_{\mathrm{ISM}} T_{\mathrm{ISM}}\right) /\left(M_{\mathrm{ICM}}+M_{\mathrm{ISM}}\right)$, which gives $M_{\mathrm{ISM}} / M_{\mathrm{ICM}}$ $\sim 8$ for $T_{\mathrm{ICM}}=6.3 \mathrm{keV}$. Thus, the cold ISM dominates the mass but resides in a much smaller volume than the hot ICM before the mixing, if pressure equilibrium is assumed. The evaporation rate of the X-ray tail by the hot ICM is unconstrained within the uncertainties, especially if the mixing is still replenishing the $\sim 0.7 \mathrm{keV}$ X-ray tail. 
As ESO 137-001 is not a massive galaxy, it should not be hard to remove its gas. According to the approximate criterion for ram pressure stripping by Gunn \& Gott (1972), at a velocity of $\sim 800 \mathrm{~km} \mathrm{~s}^{-1}$ ISM with a density of $1 \mathrm{~cm}^{-3}$ can be removed, if $n_{\mathrm{ICM}}=10^{-3} \mathrm{~cm}^{-3}$. From the approximation by Mori \& Burkert (2000), the stripping process may take $\gtrsim 10^{8} \mathrm{yr}$, if the ISM density is greater than $1 \mathrm{~cm}^{-3}$. Thus, the timescale is long enough to explain the length of the observed tail, and short enough to explain the rarity of such long X-ray tails in latetype galaxies. As there may still be unmixed cold gas in the tail and galaxy, ESO 137-001 must have been a very gas-rich galaxy, with a gas fraction of $\gtrsim 0.1$. The star formation rate in ESO 137-001 should be high, based on its very blue color and emission-line spectrum. Therefore, ESO 137-001 resembles the blue dwarf galaxies in $z \sim 0.4$ clusters that are responsible for the Butcher-Oemler effect (Butcher \& Oemler 1978). As there are no other bright cluster galaxies within $7^{\prime}$ in the tail direction, the star formation in ESO 137-001 is likely triggered by the ICM pressure (see, e.g., Vollmer et al. 2001; SV05). Thus, ESO 137-001, in its first passage through the cluster core, is being converted into a gas-poor galaxy (likely an E+A galaxy) after a possible initial starburst, all by the interaction with the dense ICM.

Tails such as that of ESO 137-001 may provide constraints on the turbulent velocity field in clusters. The ICM has long been suspected to be turbulent, although the evidence is sparse. Turbulent eddies on scales of 20-90 kpc have been reported in the Coma Cluster by Schuecker et al. (2004), who concluded that over $10 \%$ of the total ICM pressure is in turbulent form. Dolag et al. (2005) used a novel approach to treat the ICM viscosity and found that turbulent energy represents up to $8 \%$ of the thermal energy (on a scale of $70 \mathrm{kpc}$ ). If we assume that $15 \%$ of the ICM pressure is turbulent around ESO 137001 , the ICM velocity dispersion is $\sim 435 \mathrm{~km} \mathrm{~s}^{-1}$. Each part of the tail is subject to turbulent pressure in random directions, which is $2 r \rho_{\mathrm{ICM}} v_{\text {tur }}^{2}$ per unit length, where $r$ is the radius of the tail cylinder $(\sim 4 \mathrm{kpc})$ and $v_{\text {tur }}$ is the turbulent velocity. This pressure induces an acceleration $a=2 \rho_{\mathrm{ICM}} v_{\text {tur }}^{2} / \pi r \rho_{\text {tail }}$ in random directions for different parts of the tail. An eddy on a scale of $R$ lasts for a time of $\sim R / v_{\text {tur }}$, which is $\gtrsim 10^{8} \mathrm{yr}$ for $R>20 \mathrm{kpc}$. This implies that large eddies last longer than the current age of the tail. The displacement of a part of the tail over a time interval of $t$ is then $a t^{2} / 2$. The tail is straight and narrow from the end to the head, so we set an upper limit of $10^{\prime \prime}$ on the displacement. Assuming thermal pressure equilibrium between the ICM and the X-ray tail (or $\rho_{\text {tail }} / \rho_{\text {ICM }}=9$ from the temperature ratio), we have $v_{\text {tur }}<380[t /(0.05 \mathrm{Gyr})]^{-1} \mathrm{~km} \mathrm{~s}^{-1}$ on scales from $20 \mathrm{kpc}$ to the length of the tail (or $70 \mathrm{kpc}$ ). This upper limit is still consistent with the lower limit from Schuecker et al. (2004), especially if ESO 137-001 moves very fast. Turbulence can also be generated as the gas-rich ESO 137-001 moves through the ICM. However, the Reynolds number is $\sim 3 \mathcal{M}\left(L / \lambda_{i}\right)$, where $\mathcal{M}$ is the Mach number, $L$ is the size of the galaxy, and $\lambda_{i}$ is the mean free path of ions in the ICM. For unmagnetized gas, $\lambda_{i} \sim 10 \mathrm{kpc} \sim L$. Thus, unless magnetic field has reduced $\lambda_{i}$ by more than a factor of 10 , the motion of ESO 137-001 cannot drive turbulence in the trail.

Support for this work was provided by NASA grant NNG05GD52G, by NASA Long-Term Space Astrophysics grant NNG05GD82G, and by the Chandra X-Ray Center and the Smithsonian Institute.

\section{REFERENCES}

Anders, E., \& Grevesse N. 1989, Geochim. Cosmochim. Acta, 53, 197

Böhringer, H., Neumann, D. M., Schindler, S., \& Kraan-Korteweg, R. C. 1996, ApJ, 467, 168

Butcher, H., \& Oemler, A., Jr. 1978, ApJ, 219, 18

Cole, S., et al. 2001, MNRAS, 326, 255

Dolag, K., Vazza, F., Brunetti, G., \& Tormen, G. 2005, MNRAS, 364, 753

Gavazzi, G., Boselli, A., Mayer, L., Iglesias-Páramo, J., Vílchez, J. M., \& Carrasco, L. 2001, ApJ, 563, L23

Giovanelli, R., \& Haynes, M. P. 1985, ApJ, 292, 404

Gunn, J. E., \& Gott, J. R., III. 1972, ApJ, 176, 1

Jarrett, T. H. 2000, PASP, 112, 1008

Kraan-Korteweg, R. C., Woudt, P. A., Cayatte, V., Fairall, A. P., Balkowski, C., \& Henning, P. A. 1996, Nature, 379, 519

Machacek, M. E., Nulsen, P., Stirbat, L., Jones, C., \& Forman, W. R. 2005, ApJ, 630, 280
Markevitch, M., et al. 2003, ApJ, 583, 70

Mori, M., \& Burkert, A. 2000, ApJ, 538, 559

Oosterloo, T., \& van Gorkom, J. 2005, A\&A, 437, L19

Quilis, V., Moore, B., \& Bower, R. G. 2000, Science, 288, 1617

Schuecker, P., Finoguenov, A., Miniati, F., Böhringer, H., \& Briel, U. G. 2004, A\&A, 426, 387

Stevens, I. R., Acreman, D. M., \& Ponman, T. J. 1999, MNRAS, 310, 663

Sun, M., \& Vikhlinin, A. 2005, ApJ, 621, 718 (SV05)

Tamura, T., Fukazawa, Y., Kaneda, H., Makishima, K., Tashiro, M., Tanaka, Y., \& Böhringer, H. 1998, PASJ, 50, 195

Vollmer, B., Cayatte, V., Balkowski, C., \& Duschl, W. J. 2001, ApJ, 561, 708

Wang, Q. D., Owen, F., \& Ledlow, M. 2004, ApJ, 611, 821

Woudt, P. A., Kraan-Korteweg, R. C., \& Fairall, A. P. 1999, A\&A, 352, 39

Woudt, P. A., Kraan-Korteweg, R. C., Cayatte, V., Balkowski, C., \& Felenbok, P. 2004, A\&A, 415, 9

Note added in proof.-We reduced the XMM Optical Monitor (OM) data after this Letter was accepted. ESO 137-001 is a luminous galaxy in the UV band. From the OM UVW1 and UVM2 data, we derived a star formation rate of $\sim 10 M_{\odot} \mathrm{yr}^{-1}$, which makes ESO 137-001 a starburst galaxy. The starburst is most likely triggered by the interaction with the A3627 ICM. 